\title{
A COMBINATORIAL GENERALIZATION OF THE BOSON-FERMION CORRESPONDENCE
}

\author{
THOMAS LAM
}

\begin{abstract}
We attempt to explain the ubiquity of tableaux and of Pieri and Cauchy formulae for combinatorially defined families of symmetric functions. We show that such formulae are to be expected from symmetric functions arising from representations of Heisenberg algebras. The resulting framework that we describe is a generalization of the classical Boson-Fermion Correspondence, from which Schur functions arise. Our work can be used to understand Hall-Littlewood polynomials, Macdonald polynomials and Lascoux, Leclerc and Thibon's ribbon functions, together with other new families of symmetric functions.
\end{abstract}

\section{Introduction}

The classical Boson-Fermion Correspondence is an isomorphism between two representations of the Heisenberg algebra $H$ : the Bosonic Fock space $K\left[H_{-}\right]$and the Fermionic Fock space $\mathcal{F}^{(0)}$. It identifies the Schur functions $s_{\lambda}\left(x_{1}, x_{2}, \ldots\right)$ as the images of the basis of semi-infinite wedges $v_{i_{1}} \wedge v_{i_{2}} \wedge \cdots$ under this isomorphism. The Boson-Fermion Correspondence is an important basic result in mathematical physics; see for example $[7,8,11]$.

The aim of this article is to replace the classical Fermionic Fock space in the BosonFermion Correspondence by another representation of the Heisenberg algebra, and to obtain other interesting families of symmetric functions instead of the Schur functions. The symmetric functions that we obtain have a tableaux-like definition, and satisfy both Pieri-like identities and a Cauchy-like identity, which we now explain.

Let $\left\{F_{\lambda}\left(x_{1}, x_{2}, \ldots\right) \in \Lambda_{K}: \lambda \in S\right\}$ be a family of symmetric functions with coefficients in a field $K$ (usually $\mathbb{Q}, \mathbb{Q}(q)$ or $\mathbb{Q}(q, t)$ ), where $S$ is some indexing set. Many important families of symmetric functions have the following trio of properties.

(1) They can be expressed as the generating functions for a set of "tableaux", which gives the monomial expansion of $F_{\lambda}$ :

$$
F_{\lambda}\left(x_{1}, x_{2}, \ldots\right)=\sum_{T} s(T) x^{\mathrm{wt}(T)}
$$

where the sum is over tableaux $T$ with "shape" $\lambda$. The composition $\operatorname{wt}(T)$ is the weight of $T$ and $s(T) \in K$ is some additional parameter associated to $T$.

Received by the editors July 16, 2005; modified October 19, 2005.

The author was supported in part by a Clay Institute Liftoff Fellowship. 
(2) Together with a closely related dual family $\left\{G_{\lambda}\left(x_{1}, x_{2}, \ldots\right): \lambda \in S\right\}$ of symmetric functions, they satisfy a Cauchy identity:

$$
\sum_{\lambda \in S} F_{\lambda}\left(x_{1}, x_{2}, \ldots\right) G_{\lambda}\left(y_{1}, y_{2}, \ldots\right)=\prod_{i, j=1}^{\infty}\left(b_{0}+b_{1} x_{i} y_{j}+b_{2}\left(x_{i} y_{j}\right)^{2}+\cdots\right) \text {, }
$$

where the coefficients $b_{i} \in K$.

(3) They satisfy a Pieri formula:

$$
\tilde{h}_{k}\left(x_{1}, x_{2}, \ldots\right) F_{\lambda}\left(x_{1}, x_{2}, \ldots\right)=\sum_{\mu \rightarrow{ }_{k} \lambda} b_{\lambda, \mu} F_{\mu}\left(x_{1}, x_{2}, \ldots\right),
$$

where $k \in \mathbb{Z}$ is a positive integer, $\tilde{h}_{1}, \tilde{h}_{2}, \ldots \in \Lambda_{K}$ is a sequence of symmetric functions and $b_{\lambda, \mu} \in K$ are coefficients defined for each pair $\lambda, \mu$ satisfying some condition $\mu \rightarrow_{k} \lambda$.

In all such cases that the author is aware of, the definition of a tableaux involves the condition $\mu \rightarrow_{k} \lambda$ in the Pieri formula. The simplest case is when $K=\mathbb{Q}$ and $F_{\lambda}=s_{\lambda}$, the family of Schur functions. The indexing set $S=\mathcal{P}$ is the set of partitions. The tableaux are usual semi-standard Young tableaux $T$; the statistic $s(T)$ is equal to 1 and $\operatorname{wt}(T)$ is the usual weight associated to $T$. The dual family $\left\{G_{\lambda}=s_{\lambda}\right\}$ consists of the Schur functions again and in the Cauchy formula, $b_{i}=1$ for all $i$. In the Pieri formula, $\tilde{h}_{k}=h_{k}$ are the homogeneous symmetric functions. The condition $\mu \rightarrow_{k} \lambda$ is that $\mu / \lambda$ is a horizontal strip of size $k$ and $b_{\lambda, \mu}=1$. Recall in particular that a semi-standard Young tableaux is just a chain of nested partitions forming a sequence of horizontal strips.

Understanding the ubiquity of these three properties in families of symmetric functions was one of the main aims of our work. Our main result is as follows. Given a representation $V$ of a Heisenberg algebra $H$ (see Definition 1) with a distinguished basis $\left\{v_{s} \mid s \in S\right\}$, together with a highest vector $v_{b}$ in $V$, we define a family $\left\{F_{s}^{V}\left(x_{1}, x_{2}, \ldots\right) \mid s \in S\right\}$ (and a dual family $\left\{G_{s}^{V} \mid s \in S\right\}$ ) of symmetric functions which satisfy a generalized Boson-Fermion Correspondence. The definition of $F_{s}^{V}$ is tableaux-like: for example it gives the monomial expansion of $F_{s}^{V}$. We show in addition that the $F_{s}^{V}$ satisfy a Pieri rule and a Cauchy identity. Examples of symmetric functions that can be obtained in this way include the Schur functions, Schur $Q$-functions, Hall-Littlewood functions and Macdonald polynomials.

The motivating example for us is the family $\mathcal{G}_{\lambda}\left(x_{1}, x_{2}, \ldots ; q\right)$ of $q$-symmetric functions defined by Lascoux, Leclerc and Thibon [16] both combinatorially, via ribbon tableaux, and algebraically, using the action of the Heisenberg algebra on the Fock space of the quantized affine algebra $U_{q}\left(\widehat{\mathfrak{s l}}_{n}\right)$. In [14] we studied the $\mathcal{G}_{\lambda}$ in analogy with Schur functions and discovered ribbon Cauchy and Pieri identities. The current work is an attempt to understand the ribbon Cauchy and Pieri identities in a more systematic and general framework. As an application, we now give natural generalizations of the functions $\mathcal{G}_{\lambda}$ to Fock spaces of other types and also to higher level Fock spaces. By our main result, these new symmetric functions satisfy Cauchy and Pieri rules as well, and will be the subject of later work.

Our Pieri and Cauchy formulae depend heavily on a sequence $a_{i}$ of parameters appearing in the defining relations of the Heisenberg algebra $H=H\left[a_{i}\right]$ (see (5)). On the other hand, as an abstract algebra, the Heisenberg algebra does not depend on 
the choice of $a_{i}$ (as long as $a_{i} \neq 0$ ). Thus it is not clear immediately which sequences $a_{i}$ would lead to an interesting theory of symmetric functions.

Our work is also closely related to more combinatorial work of Fomin $[4,5,6]$ and of Bergeron and Sottile [1]. Fomin was mostly concerned with Schensted correspondences and Cauchy identities while Bergeron and Sottile's work has led to relations with non-commutative symmetric functions and Hopf algebras. It seems that an interesting non-commutative version of our theory also exists, though we have not attempted to make this precise in the present article.

It would be most interesting to investigate other families of symmetric functions which arise using our correspondence from other representations of Heisenberg algebras which occur naturally.

We now briefly describe the organization of the rest of the paper. In Section 2, we review the theory of Schur functions and symmetric functions. In Section 3, we describe the classical Boson-Fermion Correspondence. In Section 4, we explain how to obtain symmetric functions from representations of Heisenberg algebras. In

Section 5, we prove our generalized Boson-Fermion Correspondence. In Section 6, we prove Pieri and Cauchy formulae for our families of symmetric functions. In Section 7, we prove a partial converse to the theorems of Sections 5 and 6 . In Section 8, we give a series of examples which include Schur functions and Macdonald polynomials. We also discuss the behavior of our main construction with respect to taking direct sums or tensor products of representations. In Section 9, we explain the example of Lascoux, Leclerc and Thibon's ribbon functions studied in [16, 14]. Finally, we explain how to generalise ribbon functions to other types and higher levels, following work of Kashiwara, Miwa, Petersen and Yung [12] and Takemura and Uglov [20].

\section{Schur functions}

We will follow mostly the notation of [18]. Let $K$ be a field with characteristic 0 . Let $\Lambda_{K}$ denote the ring of symmetric functions over $K$. The ring $\Lambda_{K}$ should be thought of a subring of the ring of formal power series in countably many variables $x_{1}, x_{2}, \ldots$, of bounded degree. If the variable set is important then we write $\Lambda_{K}(X)$ or $\Lambda_{K}(Y)$. We will let $h_{1}, h_{2}, \ldots$ denote the homogeneous symmetric functions and $p_{1}, p_{2}, \ldots$ denote the power sum symmetric functions. Each of these sets forms a set of algebraically independent generators for $\Lambda_{K}$.

Let $\mathcal{P}$ denote the set of partitions. Let $\lambda=\left(\lambda_{1} \geq \lambda_{2} \geq \cdots \geq \lambda_{l}>0\right) \in \mathcal{P}$ be a partition. The size $|\lambda|$ of $\lambda$ is equal to $\lambda_{1}+\cdots+\lambda_{l}$ and we write $\lambda \vdash|\lambda|$. We also write $l(\lambda)=l$. We generally do not distinguish between a partition $\lambda$ and its Young diagram $D(\lambda)$. If $D(\mu) \subset D(\lambda)$ then $\lambda / \mu$ is a skew shape with size $|\lambda / \mu|=|\lambda|-|\mu|$.

We let $h_{\lambda}:=h_{\lambda_{1}} h_{\lambda_{2}} \cdots h_{\lambda_{l}}$ and $p_{\lambda}:=p_{\lambda_{1}} p_{\lambda_{2}} \cdots p_{\lambda_{l}}$. The sets $\left\{h_{\lambda}: \lambda \in \mathcal{P}\right\}$ and $\left\{p_{\lambda}: \lambda \in \mathcal{P}\right\}$ are bases of $\Lambda_{K}$. The homogeneous symmetric functions and the power sum symmetric functions are related by the formula

$$
h_{n}=\sum_{\lambda \vdash n} z_{\lambda}^{-1} p_{\lambda}
$$

where $z_{\lambda}=1^{m_{1}(\lambda)} m_{1}(\lambda) ! 2^{m_{2}(\lambda)} m_{2}(\lambda) ! \cdots$ and $m_{i}(\lambda)=\left|\left\{j \mid \lambda_{j}=i\right\}\right|$. 
The monomial symmetric functions are denoted $m_{\lambda}$ and the Schur functions are denoted $s_{\lambda}$. The Schur functions (and more generally skew Schur functions) are the generating functions of Young tableaux:

$$
s_{\lambda}\left(x_{1}, x_{2}, \ldots\right)=\sum_{T} x^{\mathrm{wt}(T)},
$$

where the sum is over all semistandard Young tableaux $T$ of shape $\lambda$. Alternatively, $s_{\lambda}=\sum_{\mu} K_{\lambda \mu} m_{\mu}$ where the Kostka number $K_{\lambda \mu}$ is equal to the number of semistandard Young tableaux of shape $\lambda$ and weight $\mu$. For the purposes of this paper, a Young tableaux $T$ of shape $\lambda$ should be thought of as a chain of partitions $T=\left(\emptyset=\lambda^{0} \subset\right.$ $\lambda^{1} \subset \cdots \subset \lambda^{l}=\lambda$ ) such that each skew shape $\lambda^{i} / \lambda^{i-1}$ is a horizontal strip. A horizontal strip is a skew shape containing at most one box in each column. The weight of $T$ is then the composition $\operatorname{wt}(T)=\left(\left|\lambda^{1} / \lambda^{0}\right|,\left|\lambda^{2} / \lambda^{1}\right|, \ldots,\left|\lambda^{l} / \lambda^{l-1}\right|\right)$. Similarly a Young tableaux of skew shape $\lambda / \mu$ is a chain of partitions $\left(\mu=\lambda^{0} \subset \lambda^{1} \subset \cdots \subset \lambda^{l}=\lambda\right)$.

The Schur functions satisfy the following Pieri formula, which describes how to write the product of a Schur function and a homogeneous symmetric function in terms of Schur functions:

$$
h_{k} s_{\lambda}=\sum_{\mu \rightarrow{ }_{k} \lambda} s_{\mu}
$$

where here $\mu \rightarrow_{k} \lambda$ means that the skew shape $\mu / \lambda$ is a horizontal strip of size $k$.

The Schur functions also satisfy the following Cauchy formula, which holds within the ring $\Lambda_{K}(X) \widehat{\otimes}_{K} \Lambda_{K}(Y)$, which is the completion of the tensor product of two copies of the symmetric functions.

$$
\prod_{i, j} \frac{1}{1-x_{i} y_{j}}=\sum_{\lambda} s_{\lambda}\left(x_{1}, x_{2}, \ldots\right) s_{\lambda}\left(y_{1}, y_{2}, \ldots\right)
$$

The ring of symmetric functions $\Lambda_{K}$ possesses a bilinear symmetric form $\langle.,$.$\rangle :$ $\Lambda_{K} \times \Lambda_{K} \rightarrow K$ given by $\left\langle s_{\lambda}, s_{\mu}\right\rangle=\delta_{\lambda \mu}$, or alternatively by $\left\langle p_{\lambda}, p_{\mu}\right\rangle=\delta_{\lambda \mu} z_{\lambda}$. This inner product is known as the Hall inner product. If $f \in \Lambda_{K}$ then $f^{\perp} \in \operatorname{End}\left(\Lambda_{K}\right)$ denotes the linear operator adjoint to multiplication by $f$. As a particular case $p_{k}^{\perp}=k \frac{\partial}{\partial p_{k}}$ where the differential operator acts on symmetric functions written as polynomials in the power sum symmetric functions.

\section{The classical Boson-Fermion correspondence}

Let $K$ be a field with characteristic 0 .

Definition 1. The Heisenberg algebra $H=H\left[a_{i}\right]$ is the associative algebra over $K$ with 1 generated by $\left\{B_{k}: k \in \mathbb{Z} \backslash\{0\}\right\}$ satisfying

$$
\left[B_{k}, B_{l}\right]=l \cdot a_{l} \cdot \delta_{k,-l},
$$

for some non-zero parameters $a_{l} \in K$ satisfying $a_{l}=-a_{-l}$.

As an abstract algebra, $H$ does not depend on the choice of the elements $a_{l}$, since the generators $B_{k}$ can be re-scaled to force $a_{l}=1$. However, we shall be concerned with representations of $H$, and some choices of the generators $B_{k}$ will be more natural. 
Let $K\left[H_{-}\right]=K\left[B_{-1}, B_{-2}, \ldots\right]$ denote the Bosonic Fock space representation of $H$. The action of the Heisenberg algebra on $K\left[B_{-1}, B_{-2}, \ldots\right]$ is determined by letting $B_{k}$ act by multiplication for $k<0$ and setting $B_{k} \cdot 1=0$ for $k>0$.

One can identify $K\left[B_{-1}, B_{-2}, \ldots\right]$ with the algebra $\Lambda_{K}$ of symmetric functions over $K$ by identifying $B_{-k}$ with $a_{k} p_{k}$ for $k>0$. The action of $H$ on $\Lambda_{K}$ is then given by

$$
B_{k} \longmapsto \begin{cases}a_{-k} p_{-k} & \text { for } k \leq-1, \\ k \frac{\partial}{\partial p_{k}} & \text { for } k \geq 1 .\end{cases}
$$

Let $\lambda$ be a partition. We define $B_{\lambda} \in H$ by $B_{\lambda}:=B_{\lambda_{1}} B_{\lambda_{2}} \cdots B_{\lambda_{l(\lambda)}}$. Let $D_{k}:=$ $\sum_{\lambda \vdash k} z_{\lambda}^{-1} B_{\lambda}$ where $z_{\lambda}$ is as defined in Section 2. Thus $B_{\lambda}$ and $D_{k}$ are related in the same way as $p_{\lambda}$ and $h_{k}$ (see (1)).

Similarly define $B_{-\lambda}:=B_{-\lambda_{1}} B_{-\lambda_{2}} \cdots B_{-\lambda_{l(\lambda)}}$ and $U_{k}:=\sum_{\lambda \vdash k} z_{\lambda}^{-1} B_{-\lambda}$. Also let $S_{\lambda} \in H$ be given by $S_{\lambda}:=\sum_{\mu} z_{\mu}^{-1} \chi_{\mu}^{\lambda} B_{-\mu}$ where the coefficients $\chi_{\mu}^{\lambda}$ are the characters of the symmetric group given by $s_{\lambda}=\sum_{\mu} z_{\mu}^{-1} \chi_{\mu}^{\lambda} p_{\mu}$. We will need the following standard lemma later.

Lemma 2. Let $k \geq 1$ be an integer and $\lambda$ be a partition. Then

$$
B_{-k} B_{\lambda}=k a_{k} m_{k}(\lambda) B_{\mu}+B_{\lambda} B_{-k},
$$

where $\mu$ is $\lambda$ with one less part equal to $k$. If $m_{k}(\lambda)=0$ then the first term is just 0 .

If $V$ is a representation of $H$, then a vector $v \in V$ is called a highest weight vector if $B_{k} \cdot v=0$ for $k>0$. The following result is well known. See for example [11, Proposition 2.1].

Proposition 3. Let $V$ be an irreducible representation of $H$ with non-zero highest weight vector $v \in V$. Then there exists a unique isomorphism of $H$-modules $\phi$ : $V \stackrel{\sim}{\longrightarrow} K\left[B_{-1}, B_{-2}, \ldots\right]$ such that $\phi(v)=1$.

For the remainder of this section we assume that $H=H[1]$ is given by the parameters $a_{l}=1$ for $l \geq 1$ and $a_{l}=-1$ for $l \leq-1$. Let $W=\oplus_{j \in \mathbb{Z}} K v_{j}$ be an infinite-dimensional vector space with basis $\left\{v_{j}: j \in Z\right\}$. Let $\mathcal{F}^{(0)}$ denote the vector space with basis given by semi-infinite monomials of the form $v_{i_{0}} \wedge v_{i_{-1}} \wedge \cdots$ where the indices satisfy:

(i) $i_{0}>i_{-1}>i_{-2}>\cdots$

(ii) $i_{k}=k$ for $k$ sufficiently small.

We will call $\mathcal{F}^{(0)}$ the Fermionic Fock space.

Remark 1. Usually $\mathcal{F}^{(0)}$ is considered a subspace of a larger Fock space $\mathcal{F}=\oplus_{m \in \mathbb{Z}} \mathcal{F}^{(m)}$. The spaces $\mathcal{F}^{(m)}$ are defined as for $\mathcal{F}^{(0)}$ with the condition (ii) replaced by the condition (ii $\left.{ }^{(m)}\right): i_{k}=k-m$ for $k$ sufficiently small.

Define an action of $H$ on $\mathcal{F}^{(0)}$ by

$$
B_{k} \cdot\left(v_{i_{0}} \wedge v_{i_{-1}} \wedge \cdots\right)=\sum_{j \leq 0} v_{i_{0}} \wedge v_{i_{-1}} \wedge \cdots \wedge v_{i_{j-1}} \wedge v_{i_{j}-k} \wedge v_{i_{j+1}} \wedge \cdots .
$$

The monomials are to be reordered according to the usual exterior algebra commutation rules so that $v_{i_{0}} \wedge \cdots \wedge v_{i_{j}} \wedge v_{i_{j+1}} \wedge \cdots=-v_{i_{0}} \wedge \cdots \wedge v_{i_{j+1}} \wedge v_{i_{j}} \wedge \cdots$. Thus the sum on the right hand side of (6) is actually finite so the action is well defined. One 
can check that we indeed do obtain an action of $H$. It is also not hard to see that the representation of $H$ on $\mathcal{F}^{(0)}$ is irreducible.

The vector $\bar{v}=v_{0} \wedge v_{-1} \wedge \cdots \in \mathcal{F}^{(0)}$ is a highest weight vector for this action of $H$. By Proposition 3, there exists an isomorphism $\sigma: \mathcal{F}^{(0)} \rightarrow \Lambda_{K}$ sending $\bar{v} \mapsto 1$. An algebraic version of the Boson-Fermion Correspondence identifies the image of $v_{i_{0}} \wedge v_{i_{-1}} \wedge \cdots$ under the isomorphism $\sigma$.

Theorem 4 ([11, Lecture 6]). Let $\lambda_{k}=i_{-k}+k$. Then $\sigma\left(v_{i_{0}} \wedge v_{i_{-1}} \wedge \cdots\right)=s_{\lambda}$.

In [11], this is called the "second" part of the Boson-Fermion Correspondence. The "first" part consists of identifying the image of certain vertex operators under $\sigma$. The Boson-Fermion Correspondence is a basic result in quantum field theory. It is important in the study of a family of non-linear differential equations known as the Kadomtsev-Petviashvili (KP) Hierarchy and also in the representation theory of affine algebras $[8,7]$. The relationship between vertex operators and symmetric function theory has been studied previously in $[9,10,18]$.

Our aim will be to generalize Theorem 4 to representations of Heisenberg algebras with arbitrary parameters $a_{i} \in K$. We will see that the symmetric functions that one obtains in this manner will always have a tableaux-like definition and satisfy Pieri and Cauchy identities. In our approach, we have ignored the vertex operators, but it would be interesting to see how they are related to our results.

\section{Symmetric functions from representations of Heisenberg algebras}

Let $H=H\left[a_{i}\right]$ be the Heisenberg algebra with parameters $a_{i} \in K$. Let $V$ be a representation of $H$ with distinguished basis $\left\{v_{s}: s \in S\right\}$ for some indexing set $S$. For simplicity we will assume that both $V$ and $S$ are $\mathbb{Z}$-graded so that $v_{s} \in V$ are homogeneous elements and $\operatorname{deg}\left(v_{s}\right)=\operatorname{deg}(s)$, and that each graded component of $V$ is finite-dimensional. We will also assume that the action of $H$ is graded in the sense that $\operatorname{deg}\left(B_{k}\right)=-m k$ for some $m \in \mathbb{Z} \backslash\{0\}$. Define an inner product $\langle.,\rangle:. V \times V \rightarrow K$ on $V$ by requiring that $\left\{v_{s} \mid s \in S\right\}$ forms an orthonormal basis, so that $\left\langle v_{s}, v_{s}^{\prime}\right\rangle=\delta_{s s^{\prime}}$.

Let $s, t \in S$. Define the generating functions

$$
F_{s / t}^{V}\left(x_{1}, x_{2}, \ldots\right)=F_{s / t}\left(x_{1}, x_{2}, \ldots\right):=\sum_{\alpha} x^{\alpha}\left\langle U_{\alpha_{l}} U_{\alpha_{l-1}} \cdots U_{\alpha_{1}} \cdot t, s\right\rangle,
$$

where the sum is over all compositions $\alpha=\left(\alpha_{1}, \alpha_{2}, \ldots, \alpha_{l}\right)$. Similarly define

$$
G_{s / t}^{V}\left(x_{1}, x_{2}, \ldots\right)=G_{s / t}\left(x_{1}, x_{2}, \ldots\right)=\sum_{\alpha} x^{\alpha}\left\langle D_{\alpha_{l}} D_{\alpha_{l-1}} \cdots D_{\alpha_{1}} \cdot s, t\right\rangle .
$$

Note that $F_{s / t}$ and $G_{s / t}$ are homogeneous with degree $\frac{\operatorname{deg}(s)-\operatorname{deg}(t)}{m}$. So in particular if $\frac{\operatorname{deg}(s)-\operatorname{deg}(t)}{m}$ is negative or non-integral then the generating functions are 0. For convenience we let $U_{\alpha}:=U_{\alpha_{l}} U_{\alpha_{l-1}} \cdots U_{\alpha_{1}}$ and $D_{\alpha}:=D_{\alpha_{l}} D_{\alpha_{l-1}} \cdots D_{\alpha_{1}}$.

The above definitions should be thought of as a tableaux-like definition, as the following example explains.

Example 5 (Schur functions). Let $H\left[a_{i}\right]=H[1]$ and $V=\mathcal{F}^{(0)}$. Set $S=\mathcal{P}$ and $v_{\lambda}:=v_{i_{0}} \wedge v_{i_{-1}} \wedge \cdots$, where $\lambda_{k}=i_{-k}+k$. Then we have

$$
U_{k} \cdot v_{\lambda}=\sum_{\mu \rightarrow{ }_{k} \lambda} v_{\mu}
$$


where the sum is over all horizontal strips $\mu / \lambda$ of size $k$. So the definition (7) of $F_{s / t}$ reduces to $(2)$ - the combinatorial definition of skew Schur functions in terms of Young tableaux.

The following Proposition is immediate from the definition, since $U_{k}$ commutes with $U_{l}$ and $D_{k}$ commutes with $D_{l}$ for all $k, l \in \mathbb{N}$.

Proposition 6. The generating functions $F_{s / t}$ and $G_{s / t}$ are symmetric functions.

As before, let $K\left[H_{-}\right] \subset H$ denote the subalgebra of $H$ generated by $\left\{B_{k} \mid k<0\right\}$ and similarly define $K\left[H_{+}\right] \subset H$. The definitions of $F_{s / t}$ and $G_{s / t}$ can be rephrased in terms of the Heisenberg-Cauchy elements $\Omega\left(H_{-}, X\right)$ and $\Omega\left(H_{+}, X\right)$ which lie in the completed tensor products $K\left[H_{-}\right] \widehat{\otimes} \Lambda_{K}(X)$ and $K\left[H_{+}\right] \widehat{\otimes} \Lambda_{K}(X)$ respectively:

$$
\Omega\left(H_{-}, X\right):=\sum_{\lambda} U_{\lambda} \otimes m_{\lambda}=\sum_{\lambda} z_{\lambda}^{-1} B_{-\lambda} \otimes p_{\lambda}=\sum_{\lambda} S_{\lambda} \otimes s_{\lambda} .
$$

The last two equalities follow from the classical Cauchy identity. Also define $\Omega\left(H_{+}, X\right) \in K\left[H_{+}\right] \hat{\otimes} \Lambda_{K}(X)$ by $\Omega\left(H_{+}, X\right)=\sum_{\lambda} D_{\lambda} \otimes m_{\lambda}$.

Thus for example, one has

$$
F_{s / t}\left(x_{1}, x_{2}, \ldots\right)=\left\langle\Omega\left(H_{-}, X\right) \cdot v_{t}, v_{s}\right\rangle
$$

and

$$
G_{s / t}\left(x_{1}, x_{2}, \ldots\right)=\left\langle\Omega\left(H_{+}, X\right) \cdot v_{s}, v_{t}\right\rangle .
$$

One has in particular

$$
G_{s / t}\left(x_{1}, x_{2}, \ldots\right)=\sum_{\lambda} z_{\lambda}^{-1} p_{\lambda}\left\langle B_{\lambda} \cdot v_{s}, v_{t}\right\rangle
$$

Now let $b \in S$ be such that $v_{b}$ is a highest weight vector for $H$. We will write $F_{s}:=F_{s / b}$ and $G_{s}:=G_{s / b}$. The element $\Omega\left(H_{-}, X\right) \cdot v_{b} \in V \hat{\otimes} \Lambda_{K}(X)$ depends only on the choice of $v_{b}$. The symmetric functions $F_{s}$ are the coefficients of $\Omega\left(H_{-}, X\right) \cdot v_{b}$ when it is written in the basis $\left\{v_{s} \mid s \in S\right\}$ :

$$
\Omega\left(H_{-}, X\right) \cdot v_{b}=\sum_{s} v_{s} \otimes F_{s}\left(x_{1}, x_{2}, \ldots\right) .
$$

\section{Generalization of Boson-Fermion correspondence}

Let us suppose that $b \in S$ has been picked so that $v_{b} \in V$ is a highest weight vector for $H$. By Proposition 3, there is a canonical map of $H$-modules $\phi: H \cdot b \rightarrow \Lambda_{K}$ sending $v_{b} \mapsto 1$. Our choice of inner product for $V$ allows us to give a map $\Phi: V \rightarrow \Lambda_{K}$.

Theorem 7 (Generalized Boson-Fermion Correspondence). The map $\Phi: V \rightarrow \Lambda_{K}$ given by $v_{s} \mapsto G_{s}\left(x_{1}, x_{2}, \ldots\right)$ is a map of $H$-modules.

Recall that $B_{-k}$ acts on $\Lambda_{K}$ by multiplication by $a_{k} p_{k}$ and $B_{k}$ acts as $k \frac{\partial}{\partial p_{k}}$, for $k \geq 1$.

Proof. Let us calculate $B_{l} \cdot G_{s}$ and compare with $\Phi\left(B_{l} \cdot v_{s}\right)$. Suppose first that $l<0$ and let $k=-l$. Let $\lambda$ be a partition and let $\mu$ be $\lambda$ with one less part equal to $k$. If $\lambda$ has no part equal to $k$, then $\mu$ can be any partition in the following formulae. First 
write $\left\langle B_{\lambda} B_{l} \cdot v_{s}, v_{b}\right\rangle=k a_{k} m_{k}(\lambda)\left\langle B_{\mu} v_{s}, v_{b}\right\rangle$, using a slight variation of Lemma 2 for our $H$. Alternatively, one can also compute

$$
B_{\lambda} B_{l} \cdot v_{s}=B_{\lambda} \sum_{c}\left\langle B_{l} \cdot v_{s}, v_{c}\right\rangle v_{c}=\sum_{c, d}\left\langle B_{l} \cdot v_{s}, v_{c}\right\rangle\left\langle B_{\lambda} \cdot v_{c}, v_{d}\right\rangle v_{d}
$$

so that taking the coefficient of $v_{b}$ we obtain

$$
k a_{k} m_{k}(\lambda)\left\langle B_{\mu} \cdot v_{s}, v_{b}\right\rangle=\sum_{c}\left\langle B_{l} \cdot v_{s}, v_{c}\right\rangle\left\langle B_{\lambda} \cdot v_{c}, v_{b}\right\rangle .
$$

Now,

$$
\begin{array}{rlrl}
B_{l} \cdot G_{s} & =a_{k} p_{k} G_{s} & & \text { using (8), } \\
& =a_{k} \sum_{\mu} z_{\mu}^{-1} p_{k} p_{\mu}\left\langle B_{\mu} \cdot v_{s}, v_{b}\right\rangle & \\
& =\sum_{\lambda} z_{\lambda}^{-1} p_{\lambda}\left(\sum_{c}\left\langle B_{l} \cdot v_{s}, v_{c}\right\rangle\left\langle B_{\lambda} \cdot v_{c}, v_{b}\right\rangle\right) & \text { using (9) } \\
& =\sum_{c}\left\langle B_{l} \cdot v_{s}, v_{c}\right\rangle\left(\sum_{\lambda} z_{\lambda}^{-1}\left\langle B_{\lambda} \cdot v_{c}, v_{b}\right\rangle\right) & \\
& =\sum_{c}\left\langle B_{l} \cdot v_{s}, v_{c}\right\rangle G_{c} .
\end{array}
$$

This shows that $\Phi\left(B_{l} \cdot v_{s}\right)=B_{l} \cdot \Phi\left(v_{s}\right)$ for $l<0$.

Now suppose $k>0$, and let $\lambda$ and $\mu$ be related as before. Then

$$
\begin{aligned}
B_{k} \cdot G_{s} & =k \sum_{\lambda} z_{\lambda}^{-1} \frac{\partial}{\partial p_{k}} p_{\lambda}\left\langle B_{\lambda} \cdot v_{s}, v_{b}\right\rangle \\
& =k \sum_{\lambda} z_{\lambda}^{-1} m_{k}(\lambda) p_{\mu}\left\langle B_{\mu} B_{k} \cdot v_{s}, v_{b}\right\rangle \\
& =\sum_{\mu} z_{\mu}^{-1} p_{\mu}\left\langle B_{\mu} \cdot \sum_{c}\left\langle B_{k} \cdot v_{s}, v_{c}\right\rangle v_{c}, v_{b}\right\rangle \\
& =\sum_{c}\left\langle B_{k} \cdot v_{s}, v_{c}\right\rangle\left(\sum_{\mu} z_{\mu}^{-1} p_{\mu}\left\langle B_{\mu} \cdot v_{c}, v_{b}\right\rangle\right) \\
& =\sum_{c}\left\langle B_{k} \cdot v_{s}, v_{c}\right\rangle G_{c} .
\end{aligned}
$$

This completes the proof.

When $V$ is irreducible, the map $\Phi$ does not depend on the choice of basis, but does depend on $v_{b}$. Since the degree $\operatorname{deg}\left(v_{b}\right)$ part of $V$ is one dimensional, the image of $v \in V$ is given by the coefficient of the degree $\operatorname{deg}\left(v_{b}\right)$ part of $\Omega\left(H_{+}, X\right) \cdot v$.

If $V$ is not irreducible then the map depends on the inner product $\langle.,$.$\rangle of V$ (or equivalently, the choice of orthonormal basis).

Note that a different action of $H$ on $\Lambda_{K}$ will allow us to replace the family $G_{s}$ in Theorem 7 by $F_{s}$. More precisely, one can define the adjoint action $\vartheta: H \rightarrow \operatorname{End}(V)$ of $H$ on $V$ by letting the generators $B_{k}$ act according to the formula $\left\langle\vartheta\left(B_{k}\right) \cdot v_{s^{\prime}}, v_{s}\right\rangle=$ 
$\left\langle v_{s^{\prime}}, B_{-k} \cdot v_{s}\right\rangle$. With this new representation of $H$ on $V$, the roles of $G_{s}$ and $F_{s}$ are reversed.

\section{Pieri and Cauchy identities}

Let $h_{k}\left[a_{i}\right]$ denote the image $\theta\left(h_{k}\right)$ of $h_{k}$ under the algebra homomorphism $\theta: \Lambda \rightarrow$ $\Lambda_{K}$ given by $\theta\left(p_{k}\right)=a_{k} p_{k}$. Also let $h_{k}\left\langle a_{i}\right\rangle$ denote the image $\kappa\left(h_{k}\right)$ of $h_{k}$ under the map $\kappa: \Lambda_{K} \rightarrow K$ given by $\kappa\left(p_{k}\right)=a_{k}$. Note that if all $\left\{a_{i} \mid i \geq 1\right\}$ are positive (rational) numbers then by (1) so are the numbers $h_{k}\left\langle a_{i}\right\rangle$. Let $h_{k}^{\perp}$ be the linear operator on $\Lambda_{K}$ which is adjoint to multiplication by $h_{k}$ with respect to the Hall inner product.

Theorem 8 (Generalized Pieri Rule). Let $k \geq 1$. The following identities hold in $\Lambda_{K}$ :

$$
h_{k}\left[a_{i}\right] G_{s}=\sum_{t}\left\langle U_{k} \cdot s, t\right\rangle G_{t}
$$

and

$$
h_{k}\left[a_{i}\right] F_{s}=\sum_{t}\left\langle D_{k} \cdot t, s\right\rangle F_{t}
$$

The dual identities are:

$$
h_{k}^{\perp} G_{s}=\sum_{t}\left\langle D_{k} \cdot s, t\right\rangle G_{t}
$$

and

$$
h_{k}^{\perp} F_{s}=\sum_{t}\left\langle U_{k} \cdot t, s\right\rangle F_{t} .
$$

Proof. Follows immediately from the definitions of $U_{k}, D_{k}$ and $h_{k}\left[a_{i}\right]$ together with Theorem 7 and the comments immediately after it.

Lemma 9. The following identity holds as elements of $H\left[a_{i}\right]$ :

$$
D_{b} U_{a}=\sum_{j=0}^{m} h_{j}\left\langle a_{i}\right\rangle U_{a-j} D_{b-j}
$$

where $m=\min (a, b)$.

Proof. By definition we need to show that

$$
\left(\sum_{\lambda \vdash b} z_{\lambda}^{-1} B_{\lambda}\right)\left(\sum_{\lambda \vdash a} z_{\lambda}^{-1} B_{-\lambda}\right)=\sum_{j=0}^{m} h_{j}\left\langle a_{i}\right\rangle\left(\sum_{\lambda \vdash a-j} z_{\lambda}^{-1} B_{-\lambda}\right)\left(\sum_{\lambda \vdash b-j} z_{\lambda}^{-1} B_{\lambda}\right) .
$$

Let $\mu$ and $\nu$ be partitions such that $|\mu|=a-j$ and $|\nu|=b-j$. By (1), the coefficient of $B_{-\mu} B_{\nu}$ on the right hand side is equal to $z_{\nu}^{-1} z_{\mu}^{-1} \sum_{\lambda \vdash j} z_{\lambda}^{-1} \theta\left(p_{\lambda}\right)$. Let $\rho=\lambda \cup \mu$ and $\pi=\lambda \cup \nu$. We claim that the summand $z_{\nu}^{-1} z_{\mu}^{-1} z_{\lambda}^{-1} \theta\left(p_{\lambda}\right)$ is the coefficient of $B_{-\mu} B_{\nu}$ when applying $\left[B_{k}, B_{l}\right]=k a_{k} \delta_{k,-l}$ repeatedly to $z_{\pi}^{-1} z_{\rho}^{-1} B_{\pi} B_{-\rho}$. This is a straightforward computation, counting the number of ways of picking parts from $\rho$ and $\pi$ to make the partition $\lambda$. 
In fact the relation (10), together with the relations $\left[U_{k}, U_{l}\right]=\left[D_{k}, D_{l}\right]=0$ is equivalent to the defining relations of the Heisenberg algebra $H\left[a_{i}\right]$. This is because the sets $\left\{B_{k} \mid k \neq 0\right\}$ and $\left\{U_{k} \mid k \geq 1\right\} \cup\left\{D_{k} \mid k \geq 1\right\}$ are both generators of $H\left[a_{i}\right]$.

Theorem 10 (Generalized Cauchy Identity). We have the following identity in the completion of $\Lambda_{K}(X) \otimes \Lambda_{K}(Y)$ :

$$
\sum_{s} F_{s}\left(x_{1}, x_{2}, \ldots\right) G_{s}\left(y_{1}, y_{2}, \ldots\right)=\prod_{j, k}\left(1+h_{1}\left\langle a_{i}\right\rangle x_{j} y_{k}+h_{2}\left\langle a_{i}\right\rangle\left(x_{j} y_{k}\right)^{2}+\cdots\right) .
$$

More generally, let $r, t \in S$. Then we have

$$
\begin{aligned}
& \sum_{s} F_{s / t}\left(x_{1}, x_{2}, \ldots\right) G_{s / r}\left(y_{1}, y_{2}, \ldots\right)= \\
& \quad \prod_{j, k}\left(1+h_{1}\left\langle a_{i}\right\rangle x_{j} y_{k}+h_{2}\left\langle a_{i}\right\rangle\left(x_{j} y_{k}\right)^{2}+\cdots\right) \sum_{s} F_{r / s}\left(x_{1}, \ldots\right) G_{t / s}\left(y_{1}, \ldots\right) .
\end{aligned}
$$

Proof. Let $U(x):=1+\sum_{i>0} U_{i} x^{i}$ and similarly $D(x):=1+\sum_{i>0} D_{i} x^{i}$. The identity of Lemma 9 is equivalent to

$$
D(y) U(x)=U(x) D(y)\left(1+h_{1}\left\langle a_{i}\right\rangle x y+h_{2}\left\langle a_{i}\right\rangle(x y)^{2}+\cdots\right) .
$$

Now notice that by definition we have $F_{s / t}=\left\langle\cdots U\left(x_{3}\right) U\left(x_{2}\right) U\left(x_{1}\right) \cdot v_{t}, v_{s}\right\rangle$ and $G_{s / t}=$ $\left\langle\cdots D\left(x_{3}\right) D\left(x_{2}\right) D\left(x_{1}\right) \cdot v_{s}, v_{t}\right\rangle$. The infinite products make sense since in most factors we are picking the term equal to 1 . Thus

$$
\begin{aligned}
& \sum_{s} F_{s / t}\left(x_{1}, x_{2}, \ldots\right) G_{s / r}\left(y_{1}, y_{2}, \ldots\right) \\
& =\left\langle\cdots D\left(y_{3}\right) D\left(y_{2}\right) D\left(y_{1}\right) \cdots U\left(x_{3}\right) U\left(x_{2}\right) U\left(x_{1}\right) \cdot v_{t}, v_{r}\right\rangle \\
& =\prod_{i, j \geq 1}^{\infty}\left(1+h_{1}\left\langle a_{i}\right\rangle x_{i} y_{j}+h_{2}\left\langle a_{i}\right\rangle\left(x_{i} y_{j}\right)^{2}+\cdots\right) \\
& \quad\left\langle\cdots U\left(x_{3}\right) U\left(x_{2}\right) U\left(x_{1}\right) \cdots D\left(y_{3}\right) D\left(y_{2}\right) D\left(y_{1}\right) \cdot v_{t}, v_{r}\right\rangle \\
& =\prod_{i, j \geq 1}^{\infty}\left(1+h_{1}\left\langle a_{i}\right\rangle x_{i} y_{j}+h_{2}\left\langle a_{i}\right\rangle\left(x_{i} y_{j}\right)^{2}+\cdots\right) \\
& \sum_{s} G_{t / s}\left(y_{1}, y_{2}, \ldots\right) F_{r / s}\left(x_{1}, x_{2}, \ldots\right) .
\end{aligned}
$$

These manipulations of infinite generating functions make sense since they are well defined when we restrict ourselves to a finite subset of the set of variables $\left\{x_{1}, x_{2}, \ldots, y_{1}, y_{2}, \ldots\right\}$.

Remark 2. It is not clear at this moment which sequences $a_{i}$ and which representations of $H\left[a_{i}\right]$ would lead to interesting families of symmetric functions. However, the following may be possible indications:

- Some kind of positivity for the coefficients $h_{i}\left\langle a_{i}\right\rangle$; for example if $K=\mathbb{Q}(q)$ then we may want $h_{i}\left\langle a_{i}\right\rangle$ to have positive coefficients when expanded as a power series in $q$. 
- A Pieri formula with very few non-zero or with positive coefficients. For example, we may want the coefficients $\left\langle U_{k} \cdot s, t\right\rangle$ and $\left\langle D_{k} \cdot t, s\right\rangle$ to be positive in some sense. This would imply that the definitions of $F_{t / s}$ and $G_{t / s}$ would also have a positive monomial expansion.

The results of this Section are related to results of Fomin $[4,5,6]$ and of Bergeron and Sottile [1]. Fomin studies combinatorial operators on posets and recovers Cauchy style identities similar to ours. His approach is more combinatorial and he focuses on generalizing Schensted style algorithms to these more general situations. Bergeron and Sottile have also made definitions similar to our $F_{s / t}$. Their interests have been towards aspects related to Hopf algebras and non-commutative symmetric functions; see also $[2,3]$.

Remark 3. An interesting non-commutative version of our theory may exist, where the Heisenberg algebra is replaced with an algebra $A=\left\langle B_{k} \mid k \in \mathbb{Z}-\{0\}\right\rangle$ with relations

$$
\begin{array}{rlrl}
{\left[B_{k}, B_{l}\right]} & =0 & \text { if } k \text { and } l \text { have opposite sign and } k \neq-l, \\
{\left[B_{-k}, B_{k}\right]} & =k a_{k} .
\end{array}
$$

In this case, the generating functions $F_{s / t}$ and $G_{s / t}$ will not be symmetric functions but instead be quasi-symmetric functions.

\section{A partial converse}

A partial converse to Theorems 8 and 10 exists. In other words, if a family of symmetric functions satisfies enough properties, then one can conclude that they arise from a generalized Boson-Fermion Correspondence as in Theorem 7.

Let $V$ be a $K$-vector space with a distinguished basis $\left\{v_{s}: s \in S\right\}$. In this section, suppose that $\left\{B_{k}^{\prime} \in \operatorname{End}(V): k \in \mathbb{Z} \backslash\{0\}\right\}$ are linear operators acting on $V$. Suppose further that $B_{k}$ and $B_{l}$ commute if $k$ and $l$ have the same sign. Let $D_{k}^{\prime}:=\sum_{\lambda \vdash k} z_{\lambda}^{-1} B_{\lambda}^{\prime}$ and $U_{k}^{\prime}:=\sum_{\lambda \vdash k} z_{\lambda}^{-1} B_{-\lambda}^{\prime}$. Now we can define $F_{s / t}^{\prime}\left(x_{1}, x_{2}, \ldots\right):=$ $\sum_{\alpha} x^{\alpha}\left\langle U_{\alpha_{l}}^{\prime} U_{\alpha_{l-1}}^{\prime} \cdots U_{\alpha_{1}}^{\prime} \cdot t, s\right\rangle$ and similarly for $G_{s / t}^{\prime}$.

Theorem 11. Let $\left\{a_{k} \in K \mid k \neq 0\right\}$ be a sequence of non-zero parameters satisfying $a_{k}=a_{-k}$ and suppose that $\left\{G_{s}^{\prime} \mid s \in S\right\}$ are linearly independent. Then the following are equivalent:

(1) The operators $\left\{B_{k}^{\prime}\right\}$ generate an action of the Heisenberg algebra $H\left[a_{i}\right]$ with parameters $a_{i}$.

(2) The family $\left\{G_{s}^{\prime}\right\}$ satisfies the conclusions of Theorem 8.

(3) The families $\left\{G_{s / t}^{\prime}\right\}$ and $\left\{F_{s / t}^{\prime}\right\}$ satisfy the conclusions of Theorem 10.

Proof. That (1) implies (2) and (3) is Theorems 8 and 10.

Now suppose (2) holds. Since the family $\left\{G_{s}^{\prime}\right\}$ is linearly independent, the action of $\left\{U_{k}^{\prime}, D_{k}^{\prime}\right\}$ on $V$ is isomorphic to the action of $\left\{h_{k}\left[a_{i}\right], h_{k}^{\perp}\right\}$ on $\operatorname{span}_{K}\left\{G_{s}^{\prime}\right\}$ under the isomorphism $v_{s} \mapsto G_{s}^{\prime}$. Thus the action of the operators $B_{k}^{\prime}$ on $V$ is isomorphic to the action of $\left\{\theta\left(p_{k}\right), p_{k}^{\perp}\right\}$ on $\operatorname{span}_{K}\left\{G_{s}^{\prime}\right\}$ and so generate an action of $H\left[a_{i}\right]$. Thus (2) $\Rightarrow(1)$. 
Now suppose (3) holds. Then by the argument in the proof of Theorem 10, we must have

$$
\left\langle\left(D^{\prime}(y) U^{\prime}(x)-U^{\prime}(x) D^{\prime}(y)\left(1+h_{1}\left\langle a_{i}\right\rangle x y+h_{2}\left\langle a_{i}\right\rangle(x y)^{2}+\cdots\right)\right) \cdot v_{t}, v_{r}\right\rangle=0
$$

for every $t, r \in S$. This implies that

$$
D^{\prime}(y) U^{\prime}(x)=U^{\prime}(x) D^{\prime}(y)\left(1+h_{1}\left\langle a_{i}\right\rangle x y+h_{2}\left\langle a_{i}\right\rangle(x y)^{2}+\cdots\right)
$$

so that we have

$$
D_{b}^{\prime} U_{a}^{\prime}=\sum_{j=0}^{m} h_{j}\left\langle a_{i}\right\rangle U_{a-j}^{\prime} D_{b-j}^{\prime}
$$

Now reversing the argument in the proof of Lemma 9 , we deduce that $\left[B_{k}^{\prime}, B_{l}^{\prime}\right]=$ $k a_{k} \delta_{k,-l}$. So $(3) \Rightarrow(1)$.

\section{Examples}

8.1. Schur functions. If $K=\mathbb{Q}$ and $V=\mathcal{F}^{(0)}$ and $H=H_{\text {Schur }}=H[1]$ acts as in Section 3, then Theorem 7 is just Theorem 4, where the indexing set $S$ can be identified with the set of partitions $\mathcal{P}$. In this case, the operators $B_{k}$ and $B_{-k}$ are adjoint with respect to $\langle.,$.$\rangle and so F_{\lambda}=G_{\lambda}=s_{\lambda}$ for every $\lambda$. The definition of $s_{\lambda / \mu}=F_{\lambda / \mu}$ in terms of the operators $U_{k}$ is exactly the usual combinatorial definition of skew Schur functions in terms of semistandard Young tableaux. The symmetric function $h_{k}\left[a_{i}\right]=h_{k}$ is the usual homogeneous symmetric function and the coefficients $\left\langle U_{k} \cdot \lambda, \mu\right\rangle$ are equal to 1 if $\mu / \lambda$ is a horizontal strip of size $k$ and equal to 0 otherwise. The coefficients $h_{i}\left\langle a_{i}\right\rangle$ are all equal to 1 and Theorem 10 reduces to the usual Cauchy identity.

8.2. Direct sums. Let $V_{1}$ and $V_{2}$ be two representations of $H$ with distinguished bases $\left\{v_{s_{1}}: s_{1} \in S_{1}\right\}$ and $\left\{v_{s_{2}}: s_{2} \in S_{2}\right\}$ respectively. Then $V=V_{1} \oplus V_{2}$ is a representation of $H\left[a_{i}\right]$ with distinguished basis $\left\{v_{s} \mid s \in S_{1} \amalg S_{2}\right\}$. If $s, t \in S_{i}$ for some $i$ then $F_{s / t}^{V}=F_{s / t}^{V_{i}}$ otherwise if for example $s \in S_{1}$ and $t \in S_{2}$ we have $F_{s / t}^{V}=0$. Thus the family of symmetric functions that we obtain from $H\left[a_{i}\right]$ acting on $V$ is the union of the families of symmetric functions we obtain from $V_{1}$ and $V_{2}$.

8.3. Tensor products. Let $V_{1}$ and $V_{2}$ be two representations of $H\left[a_{i}\right]$ with distinguished bases $\left\{v_{s_{1}}: s_{1} \in S_{1}\right\}$ and $\left\{v_{s_{2}}: s_{2} \in S_{2}\right\}$ respectively, as before. Then $V_{1} \otimes V_{2}$ has a distinguished basis $\left\{v_{s_{1}} \otimes v_{s_{2}} \mid s_{1} \in S_{1}\right.$ and $\left.s_{2} \in S_{2}\right\}$. Let the Heisenberg algebra $\tilde{H}:=H\left[b_{i}\right]$ with generators $\tilde{B}_{k}$, where $b_{i}=2 a_{i}$, act on $V_{1} \otimes V_{2}$ by defining the action of $\tilde{B}_{k}$ by

$$
\tilde{B}_{k} \cdot v_{1} \otimes v_{2}=\left(B_{k} \cdot v_{1}\right) \otimes v_{2}+v_{1} \cdot\left(B_{k} \cdot v_{2}\right) .
$$

This action is natural when one views $\Lambda_{K}$ as a Hopf algebra. The action of $\tilde{U}_{k}=$ $\sum_{\lambda \vdash k} z_{\lambda}^{-1} \tilde{B}_{-\lambda}$ is given by

$$
\tilde{U}_{k} \cdot v_{1} \otimes v_{2}=\sum_{i=0}^{k}\left(U_{i} \cdot v_{1}\right) \otimes\left(U_{k-i} \cdot v_{2}\right)
$$

and similarly for $\tilde{D}_{k}$. By definition, one sees that $F_{s_{1} \otimes s_{2} / t_{1} \otimes t_{2}}^{V_{1} \otimes V_{2}}=F_{s_{1} / t_{1}}^{V_{1}} F_{s_{2} / t_{2}}^{V_{2}}$ and similarly for the $G$-functions. Thus the family of symmetric functions we obtain from 
$V=V_{1} \otimes V_{2}$ are pairwise products of the symmetric functions we obtain from $V_{1}$ and $V_{2}$.

More generally, the tensor products $V_{1} \otimes \cdots \otimes V_{n}$ lead to generating functions which are products $F_{s_{1} / t_{1}}^{V_{1}} \cdots F_{s_{n} / t_{n}}^{V_{n}}$ of $n$ original generating functions. We will denote the Heisenberg algebra acting on this tensor product by $H^{(n)}:=H\left[a_{i}^{(n)}\right]$. The parameters are given by $a_{i}^{(n)}=n a_{i}$.

8.4. Macdonald polynomials. Let $K=\mathbb{Q}(q, t)$ and let $P_{\lambda}\left(x_{1}, x_{2}, \ldots ; q, t\right)$ and $Q_{\lambda}\left(x_{1}, x_{2}, \ldots ; q, t\right)$ be the Macdonald polynomials introduced in [18]. Let $\lambda=\left(\lambda_{1}, \lambda_{2}, \ldots\right)$ be a partition and $s=(i, j) \in \lambda$ be a square. Then the armlength of $s$ is given by $a_{\lambda}(s)=\lambda_{i}-j$ and the leg-length of $s$ is given by $l_{\lambda}(s)=\lambda_{j}^{\prime}-i$. Now let $s$ be any square. Define ([18, Chapter VI, (6.20)])

$$
b_{\lambda}(s)=b_{\lambda}(s ; q, t)= \begin{cases}\frac{1-q^{a_{\lambda}(s)} t^{l_{\lambda}(s)+1}}{1-q^{a_{\lambda}(s)+1} t^{l_{\lambda}(s)}} & \text { if } s \in \lambda, \\ 1 & \text { otherwise. }\end{cases}
$$

Now let $\lambda / \mu$ be a horizontal strip. Let $C_{\lambda / \mu}$ (respectively $R_{\lambda / \mu}$ ) denote the union of columns (respectively rows) that intersect $\lambda-\mu$. Define ([18, Chapter VI, (6.24)])

$$
\phi_{\lambda / \mu}=\prod_{s \in C_{\lambda / \mu}} \frac{b_{\lambda}(s)}{b_{\mu}(s)} \text { and } \psi_{\lambda / \mu}=\prod_{s \in R_{\lambda / \mu}-C_{\lambda / \mu}} \frac{b_{\mu}(s)}{b_{\lambda}(s)} .
$$

Let $V_{\text {Mac }}$ denote the vector space over $K$ with distinguished basis labeled by partitions. Define operators $\left\{U_{k}, D_{k}: k \in \mathbb{Z}_{>0}\right\}$ by:

$$
U_{k} \cdot \lambda=\sum_{\mu} \phi_{\mu / \lambda} \mu, \quad D_{k} \cdot \lambda=\sum_{\mu} \psi_{\lambda / \mu} \mu
$$

where the sums are over horizontal strips of size $|k|$. Then $Q_{\lambda / \mu}=F_{\lambda / \mu}$ and $P_{\lambda / \mu}=$ $G_{\lambda / \mu}$, so in particular the operators $\left\{U_{k} \mid k \in \mathbb{Z}_{>0}\right\}$ commute and so do the operators $\left\{D_{k} \mid k \in \mathbb{Z}_{>0}\right\}$. Now we have ([18, Ex.7.6])

$$
\begin{aligned}
\sum_{\rho} Q_{\rho / \lambda}(X ; q, t) P_{\rho / \mu}(Y ; q, t) & = \\
& \left(\sum_{\sigma} Q_{\mu / \sigma}(X ; q, t) P_{\lambda / \sigma}(Y ; q, t)\right) \prod_{i, j} \prod_{r=0}^{\infty} \frac{1-t x_{i} y_{j} q^{r}}{1-x_{i} y_{j} q^{r}} .
\end{aligned}
$$

The product $\prod_{r=0}^{\infty} \frac{1-y t q^{r}}{1-y q^{r}}$ can be written as $\sum_{n \geq 0} g_{n}(1,0,0, \ldots ; q, t) y^{n}$ where $g_{n}$ is given by $([18$, Chapter VI, (2.9)])

$$
g_{n}\left(x_{1}, x_{2}, \ldots ; q, t\right)=\sum_{\lambda \vdash n} z_{\lambda}(q, t)^{-1} p_{\lambda}\left(x_{1}, x_{2}, \ldots\right),
$$

where $z_{\lambda}(q, t)=z_{\lambda} \prod_{i=1}^{l(\lambda)} \frac{1-q^{\lambda_{i}}}{1-t^{\lambda_{i}}}$. Using Theorem 11, we see that the operators $\left\{U_{k}, D_{k} \mid k \in \mathbb{Z}_{>0}\right\}$ generate a copy of a Heisenberg algebra $H_{\mathrm{Mac}}$. A short calculation shows that the parameters $a_{k} \in \mathbb{Q}(q, t)$ of this Heisenberg algebra are given by $a_{k}=\frac{1-t^{k}}{1-q^{k}}$. The parameters $h_{k}\left\langle a_{i}\right\rangle$ are given by $h_{k}\left\langle a_{i}\right\rangle=g_{n}(1,0,0, \ldots ; q, t)=$ $n \sum_{\lambda \vdash n} z_{\lambda}(q, t)^{-1}$.

In fact Theorem 11 shows that the Pieri (and dual Pieri) rule for Macdonald polynomials is equivalent to the (generalized) Cauchy identity for Macdonald polynomials. 
Remark 4. To obtain the Hall-Littlewood functions, one can just specialize $q=0$ in the set up of this section. However, to obtain the Schur $P$ and $Q$-functions the further specialization $t=-1$ actually causes some of the $a_{i}$ to be zero. In this case, one should actually consider the subalgebra of the Heisenberg algebra generated by the generators $B_{k}$ where $k$ is odd.

\section{Ribbon functions}

Let $n \geq 1$ be a positive integer and $K=\mathbb{Q}(q)$. In [16], a family of symmetric functions $\left\{\mathcal{G}_{\lambda}^{(n)}\left(x_{1}, x_{2}, \ldots ; q\right)\right\}$ defined in terms of ribbon tableaux, called ribbon functions or LLT-polynomials, were introduced. We shall not give the precise definition here, but refer the reader to $[16,14]$. These symmetric functions arise as the polynomials $\left\{F_{s}^{\mathbf{F}}\left(x_{1}, x_{2}, \ldots\right)\right\}$ for the action of a Heisenberg algebra $H\left[a_{i}\right]$ on the level one Fock space $\mathbf{F}$ of $U_{q}\left(\widehat{\mathfrak{s l}}_{n}\right)$. This Fock space $\mathbf{F}$ has a basis $|\lambda\rangle$ naturally labeled by partitions. The parameters are given by $a_{i}=\frac{1-q^{2 n k}}{1-q^{2 k}}$ and the action of $H\left[a_{i}\right]$, commuting with the action of $U_{q}\left(\widehat{\mathfrak{s l}}_{n}\right)$, was discovered in [13]. The actions of the generators $B_{-k}$ and $B_{k}$ of this Heisenberg algebra are adjoint with respect to the inner product $\langle\mid \lambda\rangle,|\mu\rangle\rangle=\delta_{\lambda \mu}$, and so the symmetric functions $F_{\lambda}$ and $G_{\lambda}$ for this representation of $H\left[a_{i}\right]$ coincide. In [14], a ribbon Cauchy and Pieri formula for the functions $\mathcal{G}_{\lambda}^{(n)}(X ; q)$ was deduced from the action of $H\left[a_{i}\right]$ and this is a special (in fact, motivating) case for Theorems 8 and 10.

At $q=1$, the Fock space $\mathbf{F}$ for $U_{q}\left(\widehat{\mathfrak{s l}}_{n}\right)$ should be thought of as a sum of tensor products:

$$
\mathbf{F} \cong \bigoplus_{n \text {-cores }}\left(\mathcal{F}^{(0)}\right)^{\otimes n}
$$

where $\mathcal{F}^{(0)}$ is the classical Fermionic Fock space described in Section 3. Combinatorially, the decomposition (11) is given by writing a partition in terms of its $n$-core and its $n$-quotient; see [18]. As shown in subsection 8.3, the $F$-functions we obtain in this way are products of $n$ of the $F$-functions for $\mathcal{F}^{(0)}$, that is, (skew) Schur functions. This is simply the formula $\mathcal{G}_{\lambda}\left(x_{1}, x_{2}, \ldots ; 1\right)=s_{\lambda^{(0)}} s_{\lambda^{(1)}} \cdots s_{\lambda^{(n-1)}}$ observed in [16]. In fact, the $q=1$ specialization corresponds to action of the Heisenberg algebra commuting with the action of $\widehat{\mathfrak{s l}}_{n}$ on $\mathbf{F}$.

It would be interesting to see whether ribbon functions and Macdonald polynomials can be combined by finding a deformation of the action of $\left(H_{\mathrm{Mac}}\right)^{(n)}$ on $V_{\mathrm{Mac}}^{\otimes n}$.

9.1. Ribbon functions for other types and other levels. Theorem 7 allows us to define analogues of LLT's ribbon functions $\mathcal{G}^{(n)}\left(x_{1}, x_{2}, \ldots ; q\right)$ for other (quantized) Fock spaces.

Kashiwara, Miwa, Petersen and Yung [12] have defined (level one) q-deformed Fock spaces for the affine algebras $A_{n}^{(1)}, A_{2 n}^{(2)}, B_{n}^{(2)}, A_{2 n-1}^{(2)}, D_{n}^{(1)}$ and $D_{n+1}^{(2)}$, using a sophisticated construction involving perfect crystals. Let $\Phi$ denote one of these root systems and let $U_{q}(\mathfrak{g})$ be the corresponding quantum affine algebra. Let $\mathcal{F}^{\Phi}$ be the corresponding $q$-deformed Fock space of [12], which is defined over $K=\mathbb{Q}(q)$. The space $\mathcal{F}^{\Phi}$ is equipped with an action of a Heisenberg algebra $H\left[a_{i}^{\Phi}\right]$ commuting with the action of $U_{q}(\mathfrak{g})$, where the parameters $a_{i}^{\Phi}$ are calculated in [12]. The Fock space $\mathcal{F}^{\Phi}$ also has a standard basis indexed by certain semi-infinite products $b_{1} \otimes b_{2} \otimes \cdots$ of 
elements from a perfect crystal $B$ for $U_{q}(\mathfrak{g})$. We will call this indexing set $S^{\Phi}$. There is a distinguished highest weight vector $v_{b} \in \mathcal{F}^{\Phi}$ for some "bottom element" $b \in S^{\Phi}$.

Definition 12. Let $s \in S^{\Phi}$. The ribbon function of type $\Phi$ is given by $\mathcal{G}_{s}^{\Phi}=F_{s / b}^{\mathcal{F}^{\Phi}} \in$ $\Lambda_{K}$.

When $\Phi=A_{n-1}^{(1)}$, we recover LLT's ribbon functions $\mathcal{G}^{\Phi}=\mathcal{G}^{(n)}\left(x_{1}, x_{2}, \ldots ; q\right)$. The functions $\mathcal{G}^{(n)}\left(x_{1}, x_{2}, \ldots ; q\right)$ have been found to be not only interesting combinatorially (see $[14,16]$ ) but also to be related to the global basis of the Fock space and to Kazhdan-Lusztig polynomials (see [17]). One should expect the symmetric functions $\mathcal{G}_{s}^{\Phi}$ to be interesting as well. Some work in this direction can be found in [15] and will appear separately. Note that it is not known (but in some cases a conjecture) that the action of the generators $B_{k}$ and $B_{-k}$ of the Heisenberg algebra on $\mathcal{F}^{\Phi}$ are adjoint. This would imply that $F_{s / b}^{\mathcal{F}^{\Phi}}=G_{s / b}^{\mathcal{F}^{\Phi}}$.

In another direction, Takemura and Uglov [20] have studied Fock spaces $\mathbf{F}^{n, m}$ for the quantum affine algebra $U_{q}\left(\widehat{\mathfrak{s l}}_{n}\right)$ of level $m$. These Fock spaces also possess a standard basis indexed by partitions and an action of a Heisenberg algebra $H^{n, m}$ commuting with the action of $U_{q}\left(\widehat{\mathfrak{s l}}_{n}\right)$.

Definition 13. Let $\lambda \in \mathcal{P}$. The ribbon function of rank $n$ and level $m$ is given by $\mathcal{G}_{\lambda}^{(n, m)}=F_{\lambda / \emptyset}^{\mathbf{F}^{n, m}} \in \Lambda_{K}$.

We have placed the parameters $n$ and $m$ together in the notation since as explained in [20] there is a level-rank duality in this Fock space. The case $m=1$ reduces to LLT's ribbon functions: $\mathcal{G}_{\lambda}^{(n, 1)}=\mathcal{G}_{\lambda}^{(n)}$. One should expect the functions $\mathcal{G}_{\lambda}^{(n, m)}$ to be interesting as well. The parameters $a_{i}$ for $H^{n, m}$ appear to have not yet been established, though there are precise conjectures for their values.

\section{Acknowledgements}

I thank Sergey Fomin for discussions related to this work. This work is part of my Ph.D Thesis at M.I.T., written under the guidance of Richard Stanley.

\section{References}

[1] N. Bergeron and F. Sottile, Skew Schubert functions and the Pieri formula for flag manifolds, Trans. Amer. Math. Soc. 354 (2002), no. 2, 651-673.

[2] N. Bergeron, S. Mykytiuk, F. Sottile, and S. van Willigenburg, Non-commutative Pieri operators on posets, J. Combin. Theory Ser. A 91 (2000), no. 1-2, 84-110.

[3] R. Ehrenborg, On posets and Hopf algebras, Adv. in Math. 119 (1996) 1-35.

[4] S. Fomin, Duality of graded graphs, J. Algebraic Combin. 3 (1994) 357-404.

[5] _ Schensted algorithms for dual graded graphs, J. Algebraic Combin. 4 (1995) 5-45.

[6] _ Schur operators and Knuth correspondences, J. Combin. Theory Ser. A 72 (1995) $277-292$.

[7] I. Frenkel, Two constructions of affine Lie algebra representations and boson-fermion correspondence in quantum field theory, J. Funct. Anal. 44 (1981) 259-327.

[8] M. Jimbo and T. Miwa, Solitons and infinite-dimensional Lie algebras, Publ. Res. Inst. Math. Sci. 19 (1983) 943-1001.

[9] N. Jing, Vertex operators and Hall-Littlewood symmetric functions, Adv. Math. 87 (1991) 226248.

[10] _ Boson-fermion Correspondence for Hall-Littlewood polynomials, J. Math. Phys. 36 (1995) 7073-7080. 
[11] V.G. Kac and A.K. Raina, Bombay Lectures on Highest weight representations of infinite dimensional Lie algebras, Advanced Series in Mathematical Physics Vol. 2, World Scientific 1987.

[12] M. Kashiwara, T. Miwa, J.-U.H. Petersen, and C.M. Yung, Perfect crystals and q-deformed Fock spaces, Selecta Math. (N.S.) 2 (1996) 415-499.

[13] M. Kashiwara, T. Miwa, and E. Stern, Decomposition of q-deformed Fock spaces, Selecta Math. 1 (1996) 787-805.

[14] T. Lam, Ribbon tableaux and the Heisenberg algebra, Mathematische Zeitschrift 250 (2005) $685-710$.

[15] _ Combinatorics of Ribbon Tableaux, M.I.T. Ph.D. Thesis, 2005.

[16] A. Lascoux, B. Leclerc, and J.-Y. Thibon, Ribbon tableaux, Hall-Littlewood symmetric functions, quantum affine algebras, and unipotent varieties, J. Math. Phys. 38 (1997) 1041-1068.

[17] B. Leclerc and J.-Y. Thibon, Littlewood-Richardson coefficients and Kazhdan-Lusztig polynomials, Combinatorial Methods in Representation Theory, Adv. Stud. Pure Math. 28, (2000), $155-220$.

[18] I. Macdonald, Symmetric functions and Hall Polynomials, Oxford University Press, 1995.

[19] R. Stanley, Enumerative Combinatorics, Vol. 2, Cambridge University Press, 1999.

[20] K. Takemura and D. Uglov, Representations of the quantum toroidal algebra on highest weight modules of the quantum affine algebra of type $g_{N}$. Publ. RIMS, Kyoto Univ. 35 (1999), 407-450.

Department of Mathematics, M.I.T., Cambridge, MA 02139

E-mail address: thomasl@math.mit.edu 